\title{
Freiwilliges Engagement im Zeitvergleich
}

\author{
Julia Simonson, Nadiya Kelle, Corinna Kausmann \& \\ Clemens Tesch-Römer
}

\section{Kernaussagen}

Der Anteil freiwillig engagierter Menschen ist in den letzten zwanzig Jahren gestiegen. Im Jahr 2019 üben 39,7 Prozent der Personen ab 14 Jahren in Deutschland eine freiwillige Tätigkeit aus. Im Jahr 1999 waren es noch 30,9 Prozent. Die absolute Zahl der Menschen, die sich freiwillig engagieren, ist über die letzten zwanzig Jahre hinweg gestiegen. Im Jahr 2019 engagieren sich hochgerechnet 28,8 Millionen Menschen in Deutschland freiwillig. Im Jahr 1999 waren es noch 21,6 Millionen.

Während bei der Engagementquote zwischen 1999 und 2014 ein Anstieg beobachtet werden kann, ist die Quote zwischen 2014 und 2019 stabil geblieben. Im Zeitverlauf sieht man ähnlich hohe Quoten in den Jahren 1999, 2004 und 2009 (30,9 Prozent, 32,7 Prozent und 31,9 Prozent) sowie zwei ähnlich hohe Quoten in den Jahren 2014 und 2019 (40,o Prozent und 39,7 Prozent).

Der Anstieg der Engagementquote steht in Verbindung zu den gesellschaftlichen Veränderungen der letzten zwanzig Jahre; er wird aber auch von methodischen Entscheidungen beeinflusst. Gesellschaftliche Trends wie die Bildungsexpansion, die gestiegene Erwerbsbeteiligung von Frauen, die verbesserte Gesundheit Älterer und die zunehmende öffentliche Thematisierung von Engagement können sich steigernd auf die Engagementbeteiligung ausgewirkt haben. Gleichzeitig hat die im Jahr 2014 vorgenommene Anpassung bei der Engagementerfassung im Freiwilligensurvey wahrscheinlich zu dem starken Anstieg der Engagementquote zwischen 2009 und 2014 beigetragen. Das Vorgehen bei der Prüfung von Engagementangaben in den Jahren 1999 bis 2009 hat dagegen einen vermutlich bereits im ersten Jahrzehnt des Freiwilligensurvey vorhandenen Anstieg der Engagementquote verdeckt. 
Erstmals werden die Daten des Freiwilligensurveys durchgängig auch nach Bildung gewichtet. Dies ist notwendig, da Menschen mit höherer Bildung in Befragungsstudien häufig stärker vertreten sind, als es ihrem Anteil in der Bevölkerung entspricht, und gleichzeitig auch zu größeren Anteilen freiwillig engagiert sind als Menschen mit niedriger oder mittlerer Bildung. Die ausgewiesenen Engagementquoten mit der Gewichtung inklusive Bildung repräsentieren die Verhältnisse in der Gesamtbevölkerung angemessener als bisher. Sie fallen für alle Wellen geringer aus als bislang berichtet; die Differenz beträgt jeweils drei bis vier Prozentpunkte. Der Trend des Anstiegs der Engagementquote über die letzten zwanzig Jahre ändert sich durch die Hinzunahme der Bildung als Gewichtungsfaktor nicht wesentlich. 


\subsection{Einleitung}

Die Daten des Freiwilligensurveys ermöglichen es, die Entwicklung des freiwilligen Engagements seit 1999, also über einen Zeitraum von nunmehr zwanzig Jahren nachzuzeichnen. Dabei haben sich die gesellschaftlichen Rahmenbedingungen und deren Wandel in diesem Zeitraum auf das freiwillige Engagement sowie auf dessen Ausgestaltung ausgewirkt.

In den letzten Jahrzehnten vollzogen sich verschiedene gesellschaftliche Entwicklungen, die für freiwilliges Engagements in Deutschland relevant sind: eine zunehmende Gleichstellung von Frauen und Männern, die sich auch in einer gestiegenen Erwerbsbeteiligung von Frauen zeigt, die fortgesetzte Bildungsexpansion oder auch Veränderungen der Lebensphase Alter, wie etwa eine durchschnittlich verbesserte Gesundheit älterer Menschen (Tesch-Römer et al. 2017). Die zunehmende Digitalisierung in nahezu allen gesellschaftlichen Bereichen trägt dazu bei, dass digitale Instrumente der Kommunikation und Information ins freiwillige Engagement integriert werden und dass auch ganz neue Formen freiwilligen Engagements entstehen können (Deutscher Bundestag 2020). Diese gesellschaftlichen Trends können zu einer Erhöhung des Anteils freiwillig engagierter Menschen beigetragen haben.

Allerdings können sich gesellschaftliche Veränderungen auch hemmend auf die Engagemententwicklung auswirken. So könnten beispielsweise steigende Zeitrestriktionen durch zunehmende Verpflichtungen im Erwerbsleben und eine abnehmende Grenze zwischen Erwerbstätigkeit und Privatleben durch Prozesse der Arbeitsintensivierung und Digitalisierung (Meyer et al. 2019) zu einer Stagnation des Anteils freiwillig engagierter Menschen in Deutschland in den letzten Jahren geführt haben.

In diesem Kapitel sollen folgende Fragen beantwortet werden:

a) $\mathrm{Zu}$ welchem Anteil sind Menschen ab 14 Jahren in Deutschland im Jahr 2019 freiwillig engagiert?

b) Wie hat sich der Anteil freiwillig Engagierter im Zeitvergleich seit 1999 verändert?

c) $\mathrm{Zu}$ welchem Anteil sind Menschen ab 14 Jahren in Deutschland im Jahr 2019 gemeinschaftlich aktiv?

d) Wie hat sich der Anteil der gemeinschaftlich Aktiven im Zeitvergleich seit 1999 verändert?

\subsection{Daten und Indikatoren}

Die Ergebnisse des vorliegenden Kapitels beruhen auf den Daten des Deutschen Freiwilligensurveys (FWS) und werden gewichtet dargestellt. Die Gewichtung zielt darauf ab, die Repräsentativität zu verbessern, um verallgemeinerbare Aussagen treffen zu können. In den Veröffentlichungen zu den Erhebungen der Jahre 1999 bis 2014 wurden im Rahmen des Freiwilligensurveys bislang die Gewichtungsmerkmale Ge- 
schlecht, Alter, Bundesland sowie Gemeindegrößenklasse herangezogen. Diese Gewichtung wird nun um das Gewichtungsmerkmal der schulischen Bildung ergänzt. Personen aus unterschiedlichen Bildungsgruppen nehmen mit unterschiedlicher Wahrscheinlichkeit an Umfragen teil; daher sind verschiedene Bildungsgruppen in den Daten über- beziehungsweise unterrepräsentiert. Die Gewichtung inklusive schulischer Bildung wird in diesem Bericht auf die Daten aller fünf Erhebungswellen von 1999 bis 2019 angewendet. Die Engagementquoten fallen durch dieses Vorgehen für alle Erhebungswellen niedriger aus als bisher berichtet; es ändert sich somit das Niveau, der Trend des Anstiegs der Engagementquote über die letzten zwanzig Jahre bleibt jedoch im Wesentlichen bestehen. Die Gewichtung der Daten inklusive Bildung wirkt sich auch auf andere Ergebnisse aus.

Alle Ergebnisse werden auf statistische Signifikanz getestet (verwendet wird ein Signifikanzniveau von $\mathrm{p}<0,05)$. Die Prüfung auf statistische Signifikanz zeigt an, ob ein anhand der Daten des Freiwilligensurveys gefundener Unterschied mit großer Wahrscheinlichkeit auch in der Grundgesamtheit, also beispielsweise in der Wohnbevölkerung Deutschlands, vorhanden ist (statistisch signifikant) oder nur zufällig in den Daten vorliegt (statistisch nicht signifikant).

Die dargestellten Ergebnisse fußen auf der Befragung des Freiwilligensurveys 2019, die von März bis November 2019 erfolgte. Somit ist es mithilfe der Daten des Freiwilligensurveys 2019 nicht möglich, Aussagen über spätere Entwicklungen wie beispielsweise die Auswirkungen der Corona-Pandemie auf das freiwillige Engagement zu tätigen.

Im Freiwilligensurvey wird Engagement zweistufig erfasst: Im ersten Schritt wird gemeinschaftlich öffentliche Aktivität außerhalb von Beruf und Familie erfragt. Im zweiten Schritt wird Engagement erfasst, indem alle Aktiven nach der Übernahme von Aufgaben oder Arbeiten im Bereich ihrer gemeinschaftlichen Aktivität gefragt werden. Für die Ermittlung der gemeinschaftlich öffentlichen Aktivitäten werden in der Befragung vierzehn Gesellschaftsbereiche benannt, in denen man mitmachen oder sich aktiv beteiligen kann (siehe Kasten 3-1).

Das freiwillige Engagement wird im Freiwilligensurvey im zweiten Schritt dieses Verfahrens ermittelt (Kasten 3-2). Für jeden der Bereiche, für die eine Person angegeben hat, öffentlich aktiv zu sein, wird gefragt, ob die Person in den letzten zwölf Monaten dort freiwillig und unentgeltlich oder gegen geringe Aufwandsentschädigung Aufgaben oder Arbeiten übernommen hat und um welche Tätigkeiten es sich dabei konkret handelt. Abschließend wird bereichsübergreifend noch einmal nachgefragt, ob weitere noch nicht genannte Tätigkeiten ausgeübt werden.

In diesem Kapitel werden die Ergebnisse zum freiwilligen Engagement anhand der Engagementquote dargestellt. Die Engagementquote gibt den Anteil der Personen wieder, die im zweiten Teil der Abfrage (Kasten 3-2) ,ja' angegeben haben. Zusätzlich wird im Zeitvergleich auch die Aktivitätsquote dargestellt, also der Anteil der Personen, die im ersten Teil der Abfrage (Kasten 3-1) für mindestens einen der vierzehn Bereiche, ja' angegeben haben. 


\section{Kasten 3-1 Frage zur Aktivität in vierzehn gesellschaftlichen Bereichen}

Es gibt vielfältige Möglichkeiten, außerhalb von Beruf und Familie irgendwo mitzumachen, beispielsweise in einem Verein, einer Initiative, einem Projekt oder einer Selbsthilfegruppe. Ich nenne Ihnen verschiedene Bereiche, die dafür in Frage kommen. Wenn Sie an die letzten 12 Monate denken: Haben Sie sich in einem oder mehreren dieser Bereiche aktiv beteiligt? Sind Sie oder waren Sie irgendwo aktiv...

1) ... im Bereich Sport und Bewegung z. B. in einem Sportverein oder in einer Bewegungsgruppe?

2) ... im Bereich Kultur und Musik z. B. einer Theater- oder Musikgruppe, einem Chor, einer kulturellen Vereinigung oder einem Förderverein?

3) ... im Bereich Freizeit und Geselligkeit z. B. in einem Kleingartenverein oder einem Spieletreff?

4) ... im sozialen Bereich z. B. in einem Wohlfahrtsverband oder einer anderen Hilfsorganisation, in der Nachbarschaftshilfe oder einer Selbsthilfegruppe?

5) ... im Gesundheitsbereich z. B. als Helfer/in der Krankenpflege oder bei Besuchsdiensten, in einem Verband oder einer Selbsthilfegruppe?

6) ... im Bereich Schule oder Kindergarten z. B. in der Elternvertretung, der Schülervertretung oder einem Förderverein?

7) ... in der außerschulischen Jugendarbeit oder der Bildungsarbeit für Erwachsene z. B. Kinderoder Jugendgruppen betreuen oder Bildungsveranstaltungen durchführen?

8) ... im Bereich Umwelt, Naturschutz oder Tierschutz z. B. in einem entsprechenden Verband oder Projekt?

9) ... im Bereich Politik und politische Interessenvertretung z. B. in einer Partei, im Gemeinderat oder Stadtrat, in politischen Initiativen oder Solidaritätsprojekten?

10) ... im Bereich der beruflichen Interessenvertretung außerhalb des Betriebes z. B. in einer Gewerkschaft, einem Berufsverband, einer Arbeitsloseninitiative?

11) ... im kirchlichen oder religiösen Bereich z. B. in der Kirchengemeinde, einer religiösen Organisation oder einer religiösen Gemeinschaft?

12) ... im Bereich der Justiz und der Kriminalitätsprobleme z. B. als Schöffe/in oder Ehrenrichter/in, in der Betreuung von Straffälligen oder Verbrechensopfern?

13) ... im Unfall- oder Rettungsdienst oder in der freiwilligen Feuerwehr?

14) ... in einem bisher noch nicht genannten Bereich z. B. in Bürgerinitiativen oder Arbeitskreisen zur Orts- und Verkehrsentwicklung, aber auch Bürgerclubs und sonstiges, das bisher nicht genannt wurde?

\section{Kasten 3-2 Frage zum freiwilligen Engagement in vierzehn gesellschaftlichen Bereichen}

\footnotetext{
Uns interessiert nun, ob Sie in den Bereichen, in denen Sie aktiv sind, auch ehrenamtliche Tätigkeiten ausüben oder in Vereinen, Initiativen, Projekten oder Selbsthilfegruppen engagiert sind. Es geht um freiwillig übernommene Aufgaben und Arbeiten, die man unbezahlt oder gegen geringe Aufwandsentschädigung ausübt. Sie sagten, Sie sind im Bereich [Name des Bereichs] aktiv. Wenn Sie an die letzten 12 Monate denken:

Haben Sie in diesem Bereich auch Aufgaben oder Arbeiten übernommen, die Sie freiwillig oder ehrenamtlich ausüben?
} 


\subsection{Freiwilliges Engagement und Aktivität im Zeitvergleich}

\section{Der Anteil freiwillig engagierter Menschen ist in den letzten zwanzig Jahren gestiegen}

Im Jahr 2019 üben 39,7 Prozent der Personen ab 14 Jahren in Deutschland mindestens eine freiwillige Tätigkeit aus. Das entspricht hochgerechnet etwa 28,8 Millionen Menschen. Im Vergleich mit der vorherigen Erhebung des Freiwilligensurveys findet sich in den Jahren 2014 und 2019 eine Stabilität der Engagementquote. Die Anteile betragen 40,0 Prozent im Jahr 2014 und 39,7 Prozent im Jahr 2019, der Unterschied ist nicht statistisch signifikant (Abbildung 3-1).

Abbildung 3-1 Anteile freiwillig engagierter Personen im Zeitvergleich 1999-2019 (Basis: alle Befragten)

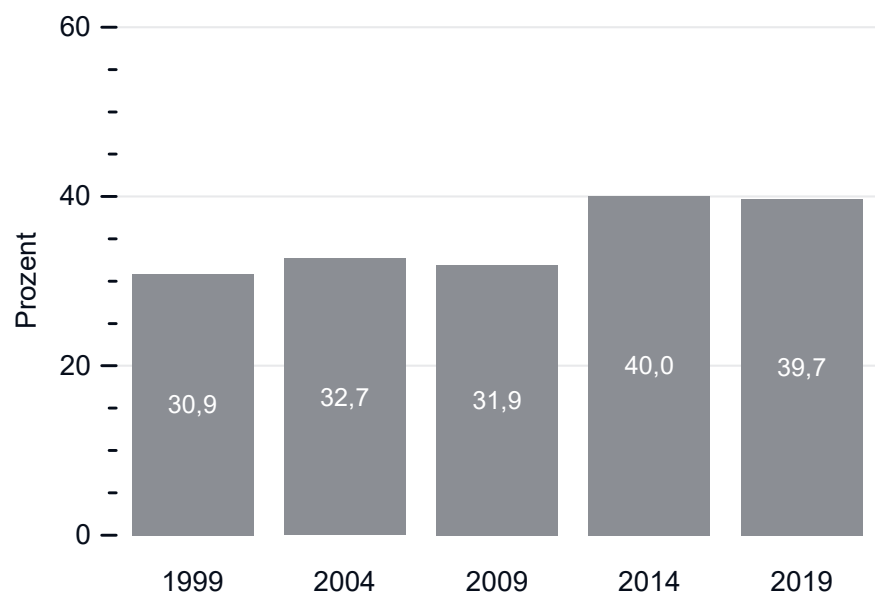

Quelle: FWS, gewichtet, eigene Berechnungen (DZA). Basis: alle Befragten, FWS 1999 ( $n=14.922)$, FWS 2004 ( $n=15.000)$, FWS 2009 $(n=20.005)$, FWS $2014(n=28.689)$, FWS $2019(n=27.759)$.

Die Unterschiede sind statistisch signifikant mit Ausnahme der Unterschiede zwischen 1999 und 2009, zwischen 2004 und 2009 sowie zwischen 2014 und 2019.

Zwischen 1999 und 2019 ist der Anteil freiwillig Engagierter gestiegen. Im Jahr 1999 übten 30,9 Prozent der Personen ab 14 Jahren in Deutschland mindestens eine freiwillige Tätigkeit aus (Abbildung 3-1). Im Jahr 2004 lag die Engagementquote bei 32,7 Prozent und im Jahr 2009 bei 31,9 Prozent. Im Jahr 2014 stieg die Engagementquote und lag bei 40,0 Prozent. Im Jahr 2019 beträgt die Engagementquote 39,7 Prozent.

Auch die absolute Anzahl der freiwillig Engagierten ist seit 1999 gestiegen. Im Jahr 2019 engagieren sich etwa 28,8 Millionen Menschen, ähnlich viele wie im Jahr 2014 
(28,4 Millionen). ${ }^{1}$ Im Jahr 1999 waren es noch 21,6 Millionen Menschen, die sich freiwillig engagierten, im Jahr 2004 waren es 23,4 Millionen Menschen und im Jahr 2009 waren es 22,8 Millionen Menschen.

Im Folgenden wird beleuchtet, welche methodischen Aspekte neben den gesellschaftlichen Veränderungen zur Erklärung des Trends der Engagementquote herangezogen werden müssen. Dabei werden die Gewichtung inklusive Bildung, die Prüfung der Angaben zum freiwilligen Engagement sowie die Anpassung der Engagementabfrage thematisiert.

\section{Der neu eingeführte Gewichtungsfaktor Bildung beeinflusst die Höhe der Engagementquoten; die Tendenz der Entwicklung des freiwilligen Engagements bleibt im Wesentlichen bestehen}

Für die Berichte zum Freiwilligensurvey 2019 werden die Daten des Freiwilligensurveys erstmals für alle Analysen auch nach Schulbildung gewichtet. Dieses Vorgehen wirkt sich somit auch auf die Höhe bislang berichteter Engagementquoten früherer Erhebungswellen aus. Die Anpassung in der Gewichtung ist notwendig, da Menschen mit höherer Bildung in Umfragestudien in der Regel stärker vertreten sind als es ihrem Anteil in der Bevölkerung entspricht. Dies gilt auch für den Freiwilligensurvey. Zugleich sind Menschen mit höherer Bildung anteilig häufiger freiwillig engagiert als Menschen mit niedriger Bildung. Ohne Berücksichtigung des Faktors Bildung bei der Gewichtung werden die Engagementquoten daher überschätzt.

Um ein angemessenes Bild des freiwilligen Engagements der Bevölkerung zu geben, müssen die Daten des Freiwilligensurvey dementsprechend nach Bildung gewichtet werden. Die anteilig stärker vertretenen Personen mit höherer Bildung werden dabei schwächer gewichtet, die anteilig schwächer vertretenen Personen mit niedriger Bildung werden dadurch stärker gewichtet. Die Engagementquoten mit Berücksichtigung des Gewichtungsfaktors Bildung fallen für alle Erhebungswellen geringer aus als die in den bisherigen Berichten dargestellten Quoten (siehe Tabelle 3-1); die Differenz beträgt jeweils drei bis vier Prozentpunkte. Die Annahmen über die Höhe der Engagementquoten müssen also korrigiert werden. Bei der Betrachtung der Engagementquoten über die Zeit ändert sich das Niveau, der Trend bleibt jedoch im Wesentlichen bestehen. Die Gewichtung der Daten inklusive Bildung wirkt sich auch auf andere Ergebnisse aus. Die Quoten mit Berücksichtigung des Gewichtungsfaktors Bildung repräsentieren die Verhältnisse in der Gesamtbevölkerung jedoch angemessener als bisher.

1 Der leichte Anstieg der absoluten Anzahl an Engagierten im Jahr 2019 im Vergleich zu 2014 begründet sich in der Bevölkerungsentwicklung Deutschlands im Vergleich der beiden Jahre: 2014 lag die Anzahl an Personen ab 14 Jahren bei rund 71,1 Millionen, 2019 hingegen bei etwa 72,5 Millionen (Statistisches Bundesamt 2020). 
Tabelle 3-1 Vergleich der Engagementquoten ohne und mit Berücksichtigung des Gewichtungsfaktors Bildung im Zeitvergleich 1999-2019

\begin{tabular}{lccccc}
\hline & FWS 1999 & FWS 2004 & FWS 2009 & FWS 2014 & FWS 2019 \\
\hline $\begin{array}{l}\text { Engagementquote ohne Berück- } \\
\text { sichtigung des Gewichtungsfak- } \\
\text { tors Bildung }\end{array}$ & 34,0 & 35,7 & 35,9 & 43,6 & 43,6 \\
$\begin{array}{l}\text { Engagementquote mit Berück- } \\
\text { sichtigung des Gewichtungsfak- } \\
\text { tors Bildung }\end{array}$ & 30,9 & 32,7 & 31,9 & 40,0 & 39,7 \\
\hline Differenz in Prozentpunkten & $-3,1$ & $-3,0$ & $-4,0$ & $-3,6$ & $-3,9$ \\
\hline
\end{tabular}

Quelle: FWS, gewichtet, eigene Berechnungen (DZA). Basis: alle Befragten. FWS $1999(n=14.922)$, FWS $2004(n=15.000)$, FWS 2009 $(n=20.005)$, FWS $2014(n=28.689)$, FWS $2019(n=27.759)$.

\section{Unterschiedliche Herangehensweisen bei der Prüfung der Angaben zum freiwilligen Engagement verdecken vermutlich einen Anstieg der Quote zwischen 1999 und 2009}

Für den Zeitraum von 1999 bis 2009 weisen die in Abbildung 3-1 dargestellten Befunde ein Plateau der Engagementquote aus. Allerdings ist aus guten Gründen anzunehmen, dass es im ersten Jahrzehnt des Freiwilligensurveys bereits einen Anstieg der Engagementquote gab, der aber aufgrund methodischer Aspekte nicht erkannt wurde. Vermutlich haben wechselnde Herangehensweisen bei der Prüfung von Engagementangaben in den Jahren 1999 bis 2009 dazu geführt, den Anstieg der Engagementquote in diesem Zeitraum zu verdecken (siehe Kapitel 2; Vogel et al. 2017). Personen, die sich in der Befragung selbst als freiwillig engagiert einschätzen, machen Angaben zu den Tätigkeiten ihres Engagements. In allen Erhebungswellen wurden diese Angaben während und nach der Befragung geprüft. Widersprechen die von der befragten Person angegebenen Informationen der Definition des freiwilligen Engagements, werden die betreffenden Tätigkeiten von ,engagiert` zu ,nicht-engagiert ${ }^{`}$ recodiert. Wenn die befragte Person insgesamt eine freiwillige Tätigkeit in der Befragung angibt und diese zu ,nicht-engagiert' recodiert wird, wird die Person dementsprechend auch als nicht-engagiert gezählt. Bei Mehrfachnennungen geschieht dies nur, wenn alle genannten Tätigkeiten von ,engagiert' zu ,nicht-engagiert' recodiert werden. Für das Jahr 2014 wurde ein Kriterienkatalog vom Deutschen Zentrum für Altersfragen (DZA) erstellt und veröffentlicht, um eine transparente Prüfung der offenen Angaben zu gewährleisten (siehe Kapitel 2). Dieser Kriterienkatalog kam auch 2019 wieder zum Einsatz.

Die Anteile der Recodierungen von, engagiert' zu ,nicht-engagiert' stiegen in den Jahren 1999 bis 2009 stetig an, ohne dass nachvollziehbar wäre, welche Gründe diesem Anstieg der Recodierungen zugrunde liegen. Dadurch wurde die Engagement- 
Tabelle 3-2 Vergleich der Engagementquoten vor und nach Prüfung der Engagementangaben im Zeitvergleich 1999-2019

\begin{tabular}{lccccc}
\hline & FWS 1999 & FWS 2004 & FWS 2009 & FWS 2014 & FWS 2019 \\
\hline Engagementquote vor Prüfung & 32,0 & 34,7 & 35,6 & 40,8 & 40,2 \\
Engagementquote nach Prüfung & 30,9 & 32,7 & 31,9 & 40,0 & 39,7 \\
\hline Differenz in Prozentpunkten & $-1,1$ & $-2,0$ & $-3,7$ & $-0,8$ & $-0,5$ \\
\hline
\end{tabular}

Quelle: FWS, gewichtet, eigene Berechnungen (DZA). Basis: alle Befragten. FWS 1999 ( $n=14.922)$, FWS 2004 ( $n=15.000$ ), FWS 2009 $(n=20.005)$, FWS $2014(n=28.689)$, FWS $2019(n=27.759)$.

quote im Verlauf der ersten zehn Jahre des Freiwilligensurveys zunehmend stärker nach unten verändert. Das lässt sich an einem Vergleich zwischen den Engagementquoten vor und nach Prüfung zeigen (siehe Tabelle 3-2). Vor Prüfung beträgt die jeweilige Engagementquote 32,o Prozent (1999), 34,7 Prozent (2004) und 35,6 Prozent (2009). Nach Prüfung beträgt die jeweilige Engagementquote 30,9 Prozent (1999), 32,7 Prozent (2004) und 31,9 Prozent (2009). Somit deuten die Anteile vor Prüfung auf einen Anstieg der Engagementquote hin, während die Anteile nach Prüfung das bereits erwähnte Plateau der Engagementquote im ersten Jahrzehnt des Freiwilligensurveys aufzeigen. Vermutlich verdecken also Vorgehensweisen bei der Prüfung der Angaben zum freiwilligen Engagement einen Anstieg der Engagementquote in den Jahren 1999 bis 2009.

\section{Die Ergänzung eines klar definierten Zeitfensters bei der Erfassung des Engagements im Jahr 2014 hat möglicherweise zum Anstieg der Engagementquote zwischen 2009 und 2014 beigetragen}

Hat es in den Jahren von 1999 bis 2009 einen Anstieg der Engagementquote gegeben, dann bedeutet dies auch, dass die Veränderung der Quote zwischen 2009 und 2014 vermutlich nicht so stark ausgeprägt ist, wie es in Abbildung 3-1 erscheint. Allerdings hat zum Anstieg der Engagementquote zwischen 2009 und 2014 möglicherweise auch die im Jahr 2014 vorgenommene Veränderung bei der Erfassung des Engagements beigetragen. Im Freiwilligensurvey 2014 wurden die Fragen zu Aktivitäten und Engagement erstmals unter Verwendung eines klar definierten Zeitfensters („,in den letzten zwölf Monaten") gestellt. In den ersten drei Erhebungswellen gab es keinen konkret vorgegebenen Zeitrahmen („derzeit“). Die Ergänzung eines klar definierten Zeitfensters sollte dazu beitragen, dass die Befragten über den gleichen zeitlichen Bezugsrahmen Auskunft geben und dass saisonales Engagement besser erfasst wird. Möglicherweise trägt die Abfrage mit dem klar definierten Zeitfenster von zwölf Monaten auch dazu bei, dass unregelmäßig ausgeübte Tätigkeiten, die ein wichtiger Bestandteil der 
Zivilgesellschaft sind, besser erfasst werden als bei der Abfrage ohne klare Zeitangabe. Unregelmäßig ausgeübte Tätigkeiten könnten dagegen unentdeckt bleiben, wenn Befragte bei einer zeitlich unspezifischen Abfrage die Zeitspannen unterschiedlich einschätzen und eher an ein kleineres Zeitfenster denken (Kelle et al. 2021).

\section{Der Anteil der Menschen, die gemeinschaftlich und öffentlich aktiv sind, ist auf hohem Niveau zwischen 1999 und 2019 leicht angestiegen}

Auch unterhalb der Schwelle freiwilligen Engagements kann man sich gesellschaftlich beteiligen und gemeinschaftlich und öffentlich aktiv sein, beispielsweise durch die Teilnahme an Sportveranstaltungen oder Freizeitaktivitäten im Verein. Die gemeinschaftlichen Aktivitäten können wiederum Anschlussmöglichkeiten zum freiwilligen Engagement bieten. Deshalb wird im Freiwilligensurvey im Vorfeld der Abfrage zum freiwilligen Engagement die Frage nach den gemeinschaftlichen Aktivitäten gestellt (siehe Kasten 3-1). Der Anteil der öffentlich gemeinschaftlich aktiven Personen - also Personen, die in mindestens einem von vierzehn im Freiwilligensurvey berücksichtigten Bereichen aktiv sind - hat sich im Zeitraum seit 1999 ebenfalls erhöht, und zwar von 62,9 Prozent im Jahr 1999 auf 66,o Prozent im Jahr 2019 (Abbildung 3-2). Allerdings zeigt sich bei dieser Entwicklung ein anderes Muster als im freiwilligen Engagement: Der Anteil an öffentlich gemeinschaftlich Aktiven lag im

Abbildung 3-2 Anteile öffentlich gemeinschaftlich aktiver Personen im Zeitvergleich 19992019 (Basis: alle Befragten)

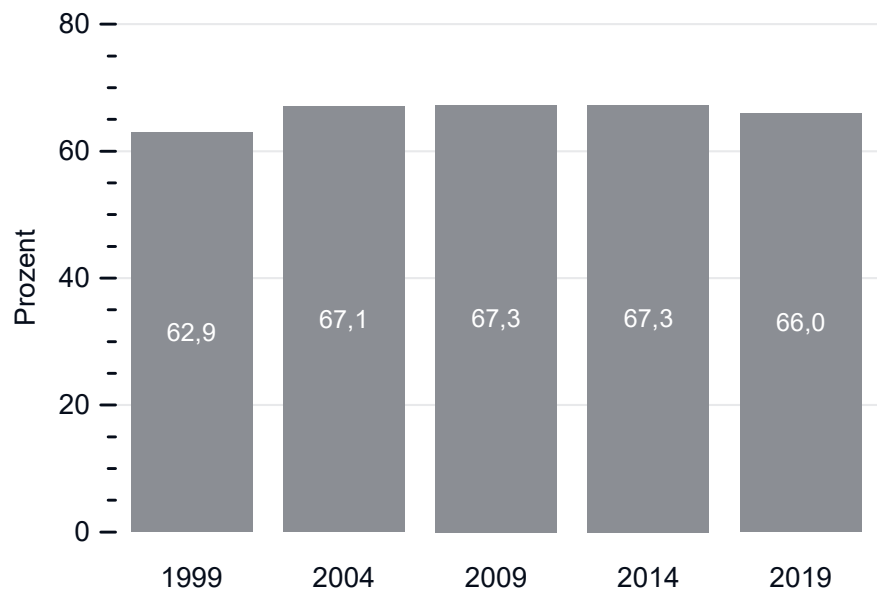

Quelle: FWS, gewichtet, eigene Berechnungen (DZA). Basis: alle Befragten, FWS 1999 ( $n=14.922)$, FWS 2004 ( $n=15.000$ ), FWS 2009 $(n=20.005)$, FWS $2014(n=28.689)$, FWS $2019(n=27.759)$.

Die Unterschiede zwischen 1999 und den drei Folgejahren (2004, 2009 und 2014) sind statistisch signifikant. Auch der Unterschied zwischen 2014 und 2019 ist statistisch signifikant. Alle restlichen Unterschiede sind nicht statistisch signifikant. 
Jahr 1999 bei 62,9 Prozent und ist im Jahr 2004 um mehr als vier Prozentpunkte gestiegen. Auch in den Folgeerhebungsjahren 2009 und 2014 blieb der Anteil Aktiver bei etwa 67 Prozent. Im Jahr 2019 ist dieser Anteil auf 66,o Prozent leicht, aber statistisch signifikant gesunken.

\subsection{Fazit}

Freiwilliges Engagement hat einen hohen Stellenwert in der Gesellschaft Deutschlands. Der Freiwilligensurvey 2019 zeigt, dass mit 39,7 Prozent etwa zwei Fünftel der Wohnbevölkerung ab 14 Jahren ein freiwilliges Engagement ausüben. Der Anteil freiwillig engagierter Menschen ist über die letzten zwei Jahrzehnte hinweg gestiegen. Zahlreiche gesellschaftliche Veränderungen, die in diesem Zeitraum stattgefunden haben, etwa die stärkere Erwerbsbeteiligung von Frauen, die fortgeführte Bildungsexpansion, die veränderte Lebenssituation älterer Menschen mit einer verbesserten gesundheitlichen Situation, die gestiegene Anzahl von Vereinen sowie die stärkere Thematisierung freiwilligen und ehrenamtlichen Engagements in der Öffentlichkeit, können zu diesem Anstieg des freiwilligen Engagements seit 1999 beigetragen haben (Tesch-Römer et al. 2017). Steigende Zeitrestriktionen durch zunehmende Verpflichtungen im Erwerbsleben und eine abnehmende Grenze zwischen Erwerbstätigkeit und Privatleben können zu der seit 2014 zu beobachtenden Stabilisierung des Anteils freiwillig engagierter Menschen in Deutschland beigetragen haben (Meyer et al. 2019; Eurofound \& International Labour Office 2017). Für einen Anstieg in den letzten zwanzig Jahren mit einer Stabilisierung auf hohem Niveau seit 2014 spricht auch, dass nicht nur der Freiwilligensurvey, sondern auch andere Umfragestudien wie der Deutsche Alterssurvey (DEAS, eigene Berechnungen) oder das Sozio-oekonomische Panel (SOEP, Burkhardt \& Schupp 2019) für den gesamten Zeitraum Zuwächse in der Engagementbeteiligung nachzeichnen, für den Zeitraum zwischen 2014 und 2019 jedoch keine wesentliche Veränderung der Engagementbeteiligung mehr feststellen. Auch die Zahl der Vereine, in denen ein großer Teil des freiwilligen Engagements stattfindet, ist nach 2014 nicht mehr so stark gestiegen wie zuvor (Priemer et al. 2019).

Allerdings zeigen die Daten des Freiwilligensurveys auch, dass die Engagementbeteiligung in den letzten zwei Jahrzehnten nicht kontinuierlich gestiegen ist, sondern dass es insbesondere zwischen 2009 und 2014 zu einem deutlichen Anstieg gekommen ist. Wenngleich es vor dem Hintergrund vielfältiger gesellschaftlicher Veränderungen durchaus plausibel ist, von einem Anstieg der Engagementbeteiligung zwischen 1999 und 2019 auszugehen, ist in Anbetracht der zu beobachtenden zwei Plateaus der Engagementquote (erstes Plateau in den Jahren 1999, 2004 und 2009, zweites Plateau in den Jahren 2014 und 2019) kritisch zu fragen, ob es sich um einen echten Anstieg handelt, der reale gesellschaftliche Veränderungen widerspiegelt, oder ob der Anstieg auch in methodischen Änderungen im Design und Vorgehen des Freiwilligensurveys begründet ist. 
Methodisch unterschiedliche Vorgehensweisen bei der Prüfung der Angaben zum freiwilligen Engagement in den Jahren 1999 bis 2009 könnten einen Anstieg der Engagementquote in diesem Zeitraum verdeckt haben. Zudem ist es möglich, dass die Veränderung der Engagementabfrage im Jahr 2014 zum Anstieg der Engagementquote zwischen 2009 und 2014 beigetragen hat. Die erstmals für alle Erhebungswellen umgesetzte Gewichtung der Daten unter Berücksichtigung des Faktors Bildung führt für alle Erhebungsjahre zu niedrigeren Engagementquoten und Hochrechnungen als in den bisherigen Berichten ausgewiesen, verändert den zu beobachtenden Trend der Engagementbeteiligung jedoch nur unwesentlich.

Welche Lehre kann aus diesen methodischen Erwägungen gezogen werden? Freiwilliges Engagement ist kein ,natürliches Phänomen', das mit einfachen Verfahren gezählt werden kann. Bei der Bestimmung des Anteils (und der Zahl) freiwillig engagierter Menschen muss zunächst definiert werden, worin freiwilliges Engagement besteht. Mit der Definition, die die Enquete-Kommission „Zukunft des bürgerschaftlichen Engagements"vorgelegt hat (Deutscher Bundestag 2002), gibt es zwar eine anerkannte Bestimmung des Konzepts, aber dennoch kann über die operationale Ausgestaltung des Begriffs in der Erfassung freiwilligen Engagements diskutiert werden. Reicht es, sich punktuell, also beispielsweise einmal pro Jahr, freiwillig zu engagieren oder muss Engagement regelmäßig und dauerhaft ausgeübt werden? Welche Tätigkeiten sind freiwilliges Engagement? Die Antworten auf diese Fragen beeinflussen die Entscheidung, ob eine Person als freiwillig engagiert eingeschätzt wird und in die Engagementquote eingeht oder nicht.

Im Jahr 2014 wurde ein Kriterienkatalog erstellt und veröffentlicht, anhand dessen die Entscheidungen, ob ein Fall als, freiwillig engagiert ' gezählt wird, nachvollziehbar und transparent gemacht wurden. Weiterhin trug die im Jahr 2014 vorgenommene Ergänzung eines Zwölf-Monats-Fensters bei der Engagementerfassung dem Umstand Rechnung, dass es saisonal und kurzfristig ausgeübte Formen des Engagements gibt. Diese können bei Abfragen, die mit dem Begriff „,derzeit“ einen unklaren, in der Wahrnehmung der Befragten aber vermutlich schmalen, Zeitrahmen vorgeben, nicht verlässlich erfasst werden. Aber auch hier gilt: Diese methodische Entscheidung, die inhaltlich sehr gut vertretbar ist, kann sich auf die Schätzung der Engagementquote auswirken (siehe Kelle et al. 2021).

Wie wird sich freiwilliges Engagement in den nächsten Jahren entwickeln? Wird die Engagementquote konstant bleiben, steigen oder möglicherweise fallen? Welchen Einfluss werden gesellschaftliche Trends und einschneidende Ereignisse wie die Corona-Pandemie haben? Diese offenen Fragen können erst in der Zukunft beantwortet werden. Dabei ist sicher, dass die Antworten auf diese Fragen vom Wirken politisch und gesellschaftlich Handelnder, aber auch von den Entscheidungen und Möglichkeiten jedes einzelnen Mitglieds der Gesellschaft abhängen. 


\section{Literatur}

Burkhardt, L., \& Schupp, J. (2019). Wachsendes ehrenamtliches Engagement: Generation der 68er häufiger auch nach dem Renteneintritt aktiv. DIW Wochenbericht Nr. 42. Online: https:// www.diw.de/documents/publikationen/73/diw_01.c.683542.de/19-42-1.pdf (zuletzt abgerufen: 10. 09. 2020).

Deutscher Bundestag (2002). Bericht der Enquete-Kommission „Zukunft des Bürgerschaftlichen Engagements“. Bürgerschaftliches Engagement: auf dem Weg in eine zukunftsfähige Bürgergesellschaft (Drucksache 14/8900). Berlin: Deutscher Bundestag.

Deutscher Bundestag (2020). Dritter Engagementbericht. Zukunft Zivilgesellschaft: Junges Engagement im digitalen Zeitalter und Stellungnahme der Bundesregierung (Drucksache 19/19320). Berlin: Deutscher Bundestag.

Eurofound \& International Labour Office (2017). Working anytime, anywhere: The effects on the world of work. Luxemburg: Publications Office of the European Union/Genf: International Labour Office.

Kelle, N., Burkhardt, L., Kausmann, C., Simonson, J., Schupp, J., \& Tesch-Römer, C. (2021). Auswirkungen von Referenzzeiträumen auf die Selbstangaben zum freiwilligen Engagement: Ergebnisse einer experimentellen Studie, SOEPpapers on Multidisciplinary Panel Data Research (Vol. 1125). Berlin: Deutsches Institut für Wirtschaftsforschung (DIW).

Meyer, S.-C., Tisch, A., \& Hünefeld, L. (2019). Arbeitsintensivierung und Handlungsspielraum in digitalisierten Arbeitswelten - Herausforderung für das Wohlbefinden von Beschäftigten? Industrielle Beziehungen: Zeitschrift für Arbeit, Organisation und Management, 26(2), 207-231.

Priemer, J., Bischoff, A., Hohendanner, C., Krebstakies, R., Rump, B., \& Schmitt, W. (2019). Organisierte Zivilgesellschaft. In: H. Krimmer (Hrsg.) Datenreport Zivilgesellschaft (S. 7-54). Wiesbaden: Springer VS.

Statistisches Bundesamt (2020). 12411-0041: Durchschnittliche Bevölkerung: Deutschland, Jahr, Nationalität, Geschlecht, Altersjahre. Online: https://www-genesis.destatis.de/genesis// online operation $=$ table $\&$ code $=12411-0041 \&$ bypass $=$ true $\&$ levelindex $=0 \&$ levelid $=16130516$ 31961\#abreadcrumb (zuletzt abgerufen 11. 02. 2021).

Tesch-Römer, C., Simonson, J., Vogel, C., \& Ziegelmann, J. P. (2017). Ergebnisse des Deutschen Freiwilligensurveys 2014: Implikationen für die Engagementpolitik. In: J. Simonson, C. Vogel \& C. Tesch-Römer (Hrsg.) Freiwilliges Engagement in Deutschland - Der Deutsche Freiwilligensurvey 2014 (S. 647-662). Wiesbaden: Springer VS.

Vogel, C., Hagen, C., Simonson, J., \& Tesch-Römer, C. (2017). Freiwilliges Engagement und öffentliche gemeinschaftliche Aktivität. In: J. Simonson, C. Vogel \& C. Tesch-Römer (Hrsg.) Freiwilliges Engagement in Deutschland - Der Deutsche Freiwilligensurvey 2014 (S. 91-151). Wiesbaden: Springer VS. 
Open Access Dieses Kapitel wird unter der Creative Commons Namensnennung Nicht kommerziell 4.0 International Lizenz (http://creativecommons.org/licenses/bync/4.0/deed.de) veröffentlicht, welche die nicht-kommerzielle Nutzung, Vervielfältigung, Bearbeitung, Verbreitung und Wiedergabe in jeglichem Medium und Format erlaubt, sofern Sie den/die ursprünglichen Autor(en) und die Quelle ordnungsgemäß nennen, einen Link zur Creative Commons Lizenz beifügen und angeben, ob Änderungen vorgenommen wurden.

Die in diesem Kapitel enthaltenen Bilder und sonstiges Drittmaterial unterliegen ebenfalls der genannten Creative Commons Lizenz, sofern sich aus der Abbildungslegende nichts anderes ergibt. Sofern das betreffende Material nicht unter der genannten Creative Commons Lizenz steht und die betreffende Handlung nicht nach gesetzlichen Vorschriften erlaubt ist, ist auch für die oben aufgeführten nicht-kommerziellen Weiterverwendungen des Materials die Einwilligung des jeweiligen Rechteinhabers einzuholen. 